\title{
Regional anesthesia and prostate cancer recurrence
}

\author{
Daniel I. Sessler, MD
}

Published online: 30 December 2009

(c) Canadian Anesthesiologists' Society 2009

Prostate cancer is the most common malignancy in men with approximately 190,000 new cases per year that kill close to 27,000 people annually in the United States alone. ${ }^{1}$ As with most cancers, treatment often involves surgical removal of the primary tumour. However, tumour surgery is usually associated with systemic release of tumour cells. Furthermore, many patients already harbour micrometastases and scattered tumour cells at the time of surgery. ${ }^{1-3}$

Whether residual cancer cells progress to clinical metastases depends largely on the balance between antimetastatic immune activity and the tumour's ability to seed, proliferate, and attract new blood vessels. ${ }^{4-6}$ At least three perioperative factors shift the balance toward progression of minimal residual disease:

- Surgery releases tumour cells into the circulation ${ }^{1-3}$; depresses cell-mediated immunity, including cytotoxic $\mathrm{T}$-cell and natural killer cell functions ${ }^{7-10}$; reduces circulating concentrations of tumour-related anti-angiogenic factors, e.g., angiostatin and endostatin ${ }^{11-14}$; increases concentrations of pro-angiogenic factors, such as vascular endothelial growth factor ${ }^{15,16}$; and releases growth factors that promote local and distant growth of malignant tissue. ${ }^{5}$

- Anesthesia impairs immune functions, including neutrophil, macrophages, dendritic cells, T-cell, and NKcell functions. ${ }^{17-20}$

- Opioids inhibit both cellular and humoral immune function in humans. ${ }^{17,21,22}$ Furthermore, morphine is

D. I. Sessler, MD ( $₫)$

Department of Outcomes Research, Anesthesiology Institute,

The Cleveland Clinic, 9500 Euclid Avenue, P77, Cleveland,

OH 44195, USA

e-mail: DS@OR.org

URL: www.or.org pro-angiogenic and promotes breast tumour growth in rodents. $^{23}$

Regional anesthesia and analgesia attenuate or prevent each of these adverse effects. For example, regional anesthesia attenuates the neuroendocrine stress response to surgery by blocking afferent neural transmission from reaching the central nervous system and activating the stress response and by blocking descending efferent activation of the sympathetic nervous system. ${ }^{24-26}$ Regional analgesia similarly reduces or eliminates the need for volatile anesthetics and opioid analgesia.

Thus, as might be expected, spinal anesthesia helps preserve natural killer cell function and reduces metastatic load to the lungs, ${ }^{7}$ and non-opioid analgesia helps preserve natural killer cell function and reduces metastatic spread of cancer in rodents. ${ }^{8,9}$ Finally, three retrospective clinical studies are consistent with this theory. (1) Melanoma recurrence was $40 \%$ more likely in patients given general rather than local anesthesia. ${ }^{27}$ (2) Paravertebral anesthesia and analgesia for breast cancer surgery were associated with approximately a four-fold reduced risk of recurrence or metastasis. ${ }^{28}$ (3) Biki et al. reported that epidural anesthesia for prostate resection halved recurrence risk. ${ }^{29}$

In this issue of the Journal, Tsui et al. evaluated biochemical recurrence after prostate cancer resection in 99 patients who were, in the context of a previous study, randomly assigned to general $v s$ epidural anesthesia more than five years ago. ${ }^{30}$ Among these patients, 22 were lost to follow-up, leaving 77 patients available for analysis.

The investigators reported no significant difference in recurrence between the two anesthetic approaches. Where

\footnotetext{
1 American Cancer Society. Cancer Facts and Figures 2009, 2009. Available from URL http://caonline.amcancersoc.org/cgi/content/full/ 59/4/225 (accessed October 2009).
} 
follow-up data were available, recurrence was observed in 11 of $35(31 \%)$ epidural patients and in 17 of $42(40 \%)$ general anesthesia patients. While these results slightly (but non-significantly) favour epidural anesthesia, log-rank testing of time-to-recurrence provided a hazard ratio of 1.3 favouring general anesthesia-but also not significantly. In fact, the $95 \%$ confidence intervals for the hazard ratio ranged widely from 0.6 to 2.7 .

There are five general threats to validity in clinical research: selection bias, measurement bias, confounding, reverse causation, and chance. Chance, i.e., statistical validity, is discussed above. Reverse causation, e.g., recurrence causing epidural anesthesia, is highly unlikely. Measurement bias is also unlikely given the objective definition of recurrence and the fact that loss to follow-up was comparably distributed between the treatment groups.

The power of randomization is that it minimizes risk from selection bias and confounding, especially confounding by unknown factors that cannot be "corrected" by multivariable statistical analysis. Thus, the great strength of Tsui et al. is random allocation of anesthetic management. However, randomization by no means assures that treatment groups are comparable-especially in small studies where, by chance, groups often differ importantly. It has thus become routine to use multivariable analysis, even in randomized trials, to statistically compensate for inter-group differences in known or suspected confounding factors. It is thus curious that Tsui et al. state that "since the subjects had been randomly allocated to anesthesia type, we did not apply adjustments for confounding." A multivariable analysis would have strengthened their results-although it surely would have left their primary conclusion intact.

In summary, the study of Tsui et al. is a retrospective analysis of a randomized trial conducted for another purpose, and because it was designed around a different endpoint, it is seriously underpowered for evaluating cancer recurrence. In contrast, Biki et al. ${ }^{29}$ included three times as many patients and obtained a statistically significant result, but the study was entirely retrospective and thus subject to the numerous and serious limitations of retrospective analyses. Both sets of investigators appropriately conclude that their respective studies do not provide anything resembling a definitive basis for concluding that regional anesthesia either is or is not protective against prostate cancer recurrence.

Tsui et al. and Biki et al. both appropriately call for large prospective trials. Clearly, large trials will be necessary to determine whether regional anesthesia-analgesia has a clinically important potential to reduce recurrence of cancer after potentially curative cancer surgery. However, prostate cancer may no longer be the right model, since long-term remission rates have improved to such an extent in recent years that a prohibitively large trial would be required. Trials should continue being conducted but might better be focused on cancers with a higher recurrence risk.

\section{L'anesthésie régionale et la récidive du cancer de la prostate}

Le cancer de la prostate est la tumeur maligne la plus courante chez les hommes; environ 190000 nouveaux cas sont dépistés chaque année, et le nombre de décès atteint près de 27000 personnes chaque année seulement aux États-Unis. ${ }^{1}$ Comme c'est le cas pour la plupart des cancers, le traitement implique souvent la résection chirurgicale de la tumeur primitive. Cependant, les chirurgies de résection de tumeur sont en général associées à une libération systémique de cellules tumorales. De plus, nombre de patients possèdent déjà des micrométastases et des cellules tumorales diffuses lors de leur chirurgie. ${ }^{1-3}$

L'évolution de cellules cancéreuses résiduelles en métastases cliniques dépend beaucoup de l'équilibre entre l'activité immunitaire anti-métastatique et la capacité de la tumeur à semer, proliférer et attirer de nouveaux vaisseaux sanguins. ${ }^{4-6}$ Au moins trois facteurs périopératoires font pencher la balance vers une progression de la maladie résiduelle minimale :

- La chirurgie libère des cellules tumorales dans la circulation $^{1-3}$; elle déprime l'immunité médiée par les cellules, y compris les fonctions des lymphocytes $\mathrm{T}$ cytotoxiques et des cellules tueuses naturelles ${ }^{7-10}$; elle réduit les concentrations en circulation de facteurs anti-angiogéniques liés à la tumeur, par ex. l'angiotensine et l'endostatine ${ }^{11-14}$; elle augmente les concentrations de facteurs pro-angiogéniques, tels que le facteur de croissance endothélial ${ }^{15,16}$; et elle libère les facteurs de croissance qui favorisent la croissance locale et distante de tissus malins. ${ }^{5}$

- L'anesthésie diminue les fonctions immunitaires, notamment les fonctions des neutrophiles, des macrophages, des cellules dendritiques, des lymphocytes $\mathrm{T}$ et des cellules tueuses naturelles. ${ }^{17-20}$

- Les opioïdes inhibent les fonctions immunitaires cellulaires et humorales chez l'humain. ${ }^{17,21,22}$ En outre, la morphine a des propriétés pro-angiogéniques et favorise la croissance de tumeur du sein chez le rongeur. ${ }^{23}$

L'anesthésie et l'analgésie régionales atténuent ou préviennent tous ces effets secondaires. Par exemple, l'anesthésie régionale atténue la réponse de stress neuroendocrinienne à la chirurgie en bloquant la transmission

\footnotetext{
${ }^{1}$ American Cancer Society. Cancer Facts and Figures 2009, 2009. Available from URL http://caonline.amcancersoc.org/cgi/content/full/ 59/4/225 (accessed October 2009).
} 
neurale afférente et en l'empêchant d'atteindre le système nerveux central, où elle activerait la réponse de stress, et en bloquant l'activation efférente descendante du système nerveux sympathique. ${ }^{24-26}$ De la même manière, l'analgésie régionale réduit ou élimine le besoin d'agents anesthésiques volatils ou d'analgésie par opioïdes.

Dès lors, comme on peut s'y attendre, la rachianesthésie aide à conserver la fonction des cellules tueuses naturelles et réduit la charge métastatique aux poumons, ${ }^{7}$ alors que l'analgésie non opioïde aide à conserver la fonction des cellules tueuses naturelles et réduit la propagation métastatique du cancer chez le rongeur. ${ }^{8,9}$ Enfin, trois études cliniques rétrospectives soutiennent cette théorie. 1) La probabilité de récidive des mélanomes était de $40 \%$ plus élevée chez les patients recevant une anesthésie générale plutôt que locale. ${ }^{27}$ 2) L'anesthésie et l'analgésie paravertébrales pour la chirurgie du cancer du sein ont été associées à une réduction par quatre environ du risque de récidive ou de métastase. ${ }^{28}$ Biki et coll. ont rapporté que l'anesthésie péridurale pour la résection de la prostate réduisait de deux fois le risque de récidive. ${ }^{29}$

Dans ce numéro du Journal, Tsui et coll. ont évalué la récidive biochimique après une résection de cancer de la prostate chez 99 patients qui, dans une étude précédente, avaient été randomisés à recevoir une anesthésie générale ou péridurale il y a plus de cinq ans. ${ }^{30}$ Parmi ces patients, 22 n'ont pas pu être suivis, ce qui laissait 77 patients dont les données pouvaient être analysées.

Les chercheurs n'ont pas rapporté de différence significative en matière de récidive entre les deux approches anesthésiques. Lorsque les données de suivi étaient disponibles, une récidive a été observée chez 11 des 35 (31\%) patients du groupe péridurale et 17 des $42(40 \%)$ patients du groupe anesthésie générale. Alors que ces résultats penchaient légèrement (mais pas significativement) en faveur de l'anesthésie péridurale, l'analyse par test logarithmique par rang a donné un taux de risque de 1,3 en faveur de l'anesthésie générale - un résultat également non significatif. En fait, les intervalles de confiance de $95 \%$ pour le taux de risque allaient de 0,6 à 2,7 , des écarts très larges.

En recherche clinique, il existe cinq menaces générales à la validité de résultats : le biais de sélection, le biais de mesure, les facteurs parasites, le lien de causalité inverse et le hasard. Le hasard, soit la validité statistique, a été discuté ci-dessus. Le lien de causalité inverse, par exemple la récidive provoquant une analgésie péridurale, est fort peu probable. Le biais de mesure est également peu probable étant donné la définition objective de la récidive et le fait que le nombre de patients n'ayant pas fait l'objet de suivi était distribué de façon comparable entre les deux groupes de traitement.
La puissance de la randomisation réside dans le fait qu'elle minimise le risque de biais de sélection et de facteurs parasites, particulièrement la confusion liée à des facteurs inconnus qui ne peuvent être «corrigés » par une analyse statistique multivariée. Dès lors, la principale force de l'étude de Tsui et coll. se trouve dans l'attribution aléatoire de la prise en charge anesthésique. Toutefois, la randomisation ne garantit en aucun cas que les groupes de traitement sont comparables - particulièrement dans des études de petite taille dans lesquelles, par hasard, les groupes comportent souvent d'importantes différences. Pour pallier à ces éléments, le recours à l'analyse multivariée est donc devenu fréquent et ce, même dans les études randomisées. En effet, une telle analyse permet de compenser statistiquement les différences entre groupes en matière de facteurs parasites connus ou soupçonnés. Dès lors, il est d'autant plus curieux que Tsui et coll. déclarent que « étant donné que les sujets ont été randomisés à recevoir un type d'anesthésie, nous n'avons pas eu recours à des ajustements pour contrer les facteurs parasites ". Une analyse multivariée aurait renforcé leurs résultats et ce, tout en gardant probablement intacte leur conclusion première.

En résumé, l'étude de Tsui et coll. consiste en l'analyse rétrospective d'une étude randomisée réalisée dans un autre but, et parce qu'elle a été conçue autour d'un critère d'évaluation différent, elle manque sérieusement de puissance quand il s'agit d'évaluer la récidive du cancer. Par contre, Biki et coll. ${ }^{29}$ ont inclus trois fois plus de patients que Tsui et obtenu un résultat significatif d'un point de vue statistique, mais leur étude était entièrement rétrospective et donc sujette aux limites à la fois nombreuses et considérables des analyses rétrospectives. Ces deux équipes de chercheurs concluent de façon adéquate que leurs études respectives n'offrent rien qui puisse être considéré comme des résultats définitifs permettant de conclure que l'anesthésie régionale protège ou non contre la récidive du cancer de la prostate.

Tsui et coll. et Biki et coll. appellent tous deux à la réalisation d'études prospectives d'envergure. Il est évident que des études de taille importante seront nécessaires afin de déterminer si l'anesthésie et l'analgésie régionales possèdent un potentiel cliniquement important de réduction de la récidive d'un cancer après une chirurgie potentiellement curative du cancer. Toutefois, il se peut que le cancer de la prostate ne soit plus le bon modèle à étudier, étant donné que les taux de rémission à long terme se sont tellement améliorés ces dernières années qu'une étude d'une taille démesurée serait requise. Il faut continuer de réaliser des études, mais elles seraient peut-être plus utiles si elles se concentraient sur des cancers présentant un risque plus élevé de récidive. 
Conflicts of interest Dr. Sessler has no personal financial interests related to this editorial.

\section{References}

1. Eschwege $P$, Dumas $F$, Blanchet $P$, et al. Haematogenous dissemination of prostatic epithelial cells during radical prostatectomy. Lancet 1995; 346: 1528-30.

2. Foss OP, Brennhovd IO, Messelt OT, Efskind J, Liverud K. Invasion of tumor cells into the bloodstream caused by palpation or biopsy of the tumor. Surgery 1966; 59: 691-5.

3. Denis MG, Lipart C, Leborgne J, et al. Detection of disseminated tumor cells in peripheral blood of colorectal cancer patients. Int J Cancer 1997; 74: 540-4.

4. Holmgren L, O'Reilly MS, Folkman J. Dormancy of micrometastases: balanced proliferation and apoptosis in the presence of angiogenesis suppression. Nat Med 1995; 1: 149-53.

5. Shakhar G, Ben-Eliyahu S. Potential prophylactic measures against postoperative immunosuppression: could they reduce recurrence rates in oncological patients? Ann Surg Oncol 2003; 10: 972-92.

6. Smyth MJ, Godfrey DI, Trapani JA. A fresh look at tumor immunosurveillance and immunotherapy. Nat Immunol 2001; 2: 293-9.

7. Bar-Yosef S, Melamed R, Page GG, Shakhar G, Shakhar K, BenEliyahu $S$. Attenuation of the tumor-promoting effect of surgery by spinal blockade in rats. Anesthesiology 2001; 94: 1066-73.

8. Ben-Eliyahu S, Page GG, Yirmiya R, Shakhar G. Evidence that stress and surgical interventions promote tumor development by suppressing natural killer cell activity. Int J Cancer 1999; 80: $880-8$.

9. Page GG, Blakely WP, Ben-Eliyahu S. Evidence that postoperative pain is a mediator of the tumor-promoting effects of surgery in rats. Pain 2001; 90: 191-9.

10. Wong IH, Lau WY, Leung T, Yeo W, Johnson PJ. Hematogenous dissemination of hepatocytes and tumor cells after surgical resection of hepatocellular carcinoma: a quantitative analysis. Clin Cancer Res 1999; 5: 4021-7.

11. Zetter BR. Angiogenesis and tumor metastasis. Annu Rev Med 1998; 49: 407-24.

12. O'Reilly MS, Holmgren L, Shing $Y$, et al. Angiostatin: a novel angiogenesis inhibitor that mediates the suppression of metastases by a Lewis lung carcinoma. Cell 1994; 79: 315-28.

13. O'Reilly MS, Boehm T, Shing Y, et al. Endostatin: an endogenous inhibitor of angiogenesis and tumor growth. Cell 1997; 88: 277-85.

14. Folkman J. Tumor angiogenesis. In: Mendelsohn J, Howley PM, Israel MA, Liotta LA, editors. The Molecular Basis of Cancer. WB Saunders; 1995

15. Lutgendorf SK, Cole S, Costanzo E, et al. Stress-related mediators stimulate vascular endothelial growth factor secretion by two ovarian cancer cell lines. Clin Cancer Res 2003; 9: 4514-21.
16. Antoni MH, Lutgendorf SK, Cole SW, et al. The influence of biobehavioural factors on tumour biology: pathways and mechanisms. Nat Rev Cancer 2006; 6: 240-8.

17. Sacerdote P, Bianchi M, Gaspani L, et al. The effects of tramadol and morphine on immune responses and pain after surgery in cancer patients. Anesth Analg 2000; 90: 1411-4.

18. Brand JM, Kirchner H, Poppe C, Schmucker P. The effects of general anesthesia on human peripheral immune cell distribution and cytokine production. Clin Immunol Immunopathol 1997; 83: $190-4$.

19. Markovic SN, Knight PR, Murasko DM. Inhibition of interferon stimulation of natural killer cell activity in mice anesthetized with halothane or isoflurane. Anesthesiology 1993; 78: 700-6.

20. Shapiro J, Jersky J, Katzav S, Feldman M, Segal S. Anesthetic drugs accelerate the progression of postoperative metastases of mouse tumors. J Clin Invest 1981; 68: 678-85.

21. Beilin B, Shavit Y, Hart J, et al. Effects of anesthesia based on large versus small doses of fentanyl on natural killer cell cytotoxicity in the perioperative period. Anesth Analg 1996; 82: 492-7.

22. Yeager MP, Colacchio TA, Yu CT, et al. Morphine inhibits spontaneous and cytokine-enhanced natural killer cell cytotoxicity in volunteers. Anesthesiology 1995; 83: 500-8.

23. Gupta K, Kshirsagar S, Chang L, et al. Morphine stimulates angiogenesis by activating proangiogenic and survival-promoting signaling and promotes breast tumor growth. Cancer Res 2002; 62: 4491-8.

24. Kehlet $H$. The stress response to surgery. In: Cousins MJ, Brindenbaugh PO, editors. Neural Blockade in Clinical Anesthesia and Management of Pain. New York: Lippincott Williams \& Wilkins; 1998 .

25. Buggy DJ, Smith G. Epidural anaesthesia and analgesia: better outcome after major surgery? Growing evidence suggests so. BMJ 1999; 319: 530-1.

26. O'Riain SC, Buggy DJ, Kerin MJ, Watson RW, Moriarty DC. Inhibition of the stress response to breast cancer surgery by regional anesthesia and analgesia does not affect vascular endothelial growth factor and prostaglandin E2. Anesth Analg 2005; 100: 244-9.

27. Schlagenhauff B, Ellwanger $U$, Breuninger $H$, Stroebel $W$, Rassner $G$, Garbe $C$. Prognostic impact of the type of anaesthesia used during the excision of primary cutaneous melanoma. Melanoma Res 2000; 10: 165-9.

28. Exadaktylos AK, Buggy DJ, Moriarty DC, Mascha E, Sessler DI. Can anesthetic technique for primary breast cancer surgery affect recurrence or metastasis? Anesthesiology 2006; 4: 660-4.

29. Biki B, Mascha E, Moriarty DC, Fitzpatrick JM, Sessler DI, Buggy DJ. Anesthetic technique for radical prostatectomy surgery affects cancer recurrence: a retrospective analysis. Anesthesiology 2008; 109: 180-7.

30. Tsui BCH, Rashiq S, Schopflocher D, Murtha A, Broemling S, Pillay J, Finucane BT. Epidural anesthesia and cancer recurrence rates after radical prostatectomy. Can J Anesth 2010; 57: XXX. 